\title{
EFFECTS OF THERMOREGULATION ON HUMAN SLEEP PATTERNS: A MATHEMATICAL MODEL OF SLEEP/WAKE CYCLES WITH REM/NREM SUBCIRCUIT
}

\author{
SELENNE BANUELOS*, JANET BEST ${ }^{\dagger}$, GEMMA HUGUET ${ }^{\ddagger}$, ALICIA \\ PRIETO-LANGARICA ${ }^{\S}$, PAMELA B. PYZZA $\uparrow$, MARKUS H. SCHMIDT", AND \\ SHELBY WILSON**
}

\begin{abstract}
In this paper we construct a mathematical model of human sleep/wake regulation with thermoregulation and temperature effects. Simulations of this model show features previously presented in experimental data such as elongation of duration and number of REM bouts across the night as well as the appearance of awakenings due to deviations in body temperature from thermoneutrality. This model helps to demonstrate the importance of temperature in the sleep cycle. Further modifications of the model to include more temperature effects on other aspects of sleep regulation such as sleep and REM latency are discussed.
\end{abstract}

Key words. REM Cycle, Sleep, Thermoregulation.

AMS(MOS) subject classifications. Primary, 92B20, 92C20.

1. Introduction. Humans split their days between waking and sleeping, spending nearly one third of life asleep. Although much progress has been made in identifying specific regions of the brain involved in regulating sleep and tracing connections between these regions, many questions remain concerning how physiological constraints are instantiated and affect the regulation of sleep and wake. Indeed, there is a lack of consensus regarding the fundamental function of sleep and which physiological needs are of primary importance in determining the structure of sleep-wake cycling $[15,41,42]$. Investigating how sleep is altered by perturbations in physiologically-relevant variables, such as temperature, has a long history (reviewed in [17]) and may shed light on how some physiological constraints shape sleep bouts. Understanding mechanisms in the brain that control sleep and wakefulness is increasingly important, as sleep disorders become more widespread in modern society [4].

During wakefulness, the brain and skeletal muscles are in a state of high metabolic activity: wakefulness is characterized by low-voltage, fast

\footnotetext{
*Department of Mathematics, California State University-Channel Islands, Camarillo, CA

${ }^{\dagger}$ Department of Mathematics, The Ohio State University, Columbus, OH

¥Departament de Matemàtica Aplicada I, Universitat Politècnica de Catalunya, Barcelona, Spain

$\S$ Department of Mathematics and Statistics, Youngstown State University, Youngstown, $\mathrm{OH}$

I Department of Mathematical Sciences, Rensselaer Polytechnic Institute, Troy, NY

\| Ohio Sleep Medicine Institute, Dublin, OH

** Department of Mathematics, Morehouse College, Atlanta, GA
} 
electroencephalogram (EEG) activity and high muscle tone. Sleep can be broadly classified as rapid eye movement (REM) sleep, during which the level of brain metabolic activity (and resulting EEG) are similar to that of wakefulness but there is an absence of skeletal muscle tone, and non-rapid eye movement (NREM) sleep, characterized by high amplitude, low-frequency EEG and an intermediate level of muscle tone $[3,8]$. During NREM sleep, often consider the most restful state; the brain has a decreased level of metabolic activity; the power of low-frequency $(0-4 \mathrm{~Hz})$ activity in the EEG is regarded as a measure of the depth or restfulness of sleep.

The timing, depth, and duration of sleep are regulated by the circadian (approximately 24-hour) rhythm and an appetitive or homeostatic drive for sleep following a period of wakefulness [9, 8]. A typical night of sleep begins with NREM sleep, then alternates between NREM and REM sleep over the course of the night [14] with REM episodes usually becoming longer across the night. The average period of the NREM-REM cycle is approximately 90-110 minutes [25].

The focus of this paper is the relationship between sleep and temperature. As endotherms, humans have the ability to maintain a metabolically favorable core body temperature $(\mathrm{CBT})$ of approximately $37^{\circ} \mathrm{C}$ over a wide range of ambient temperatures. The human body uses a variety of processes to adjust CBT such as intracorporal transport of heat by blood circulation [19], shivering, sweating, vasodilatation and vasoconstriction, as well as changing body position. Ambient temperatures that require only very subtle changes in cutaneous blood flow to maintain CBT are referred to as "thermoneutral" [22]. There is a circadian rhythm of CBT with an amplitude of about $0.5^{\circ} \mathrm{C}$, and humans typically fall asleep during the descending phase of the temperature rhythm $[10,43,44]$.

Experiments in humans have shown that, during sleep, our thermoregulatory abilities are decreased (NREM) or nearly absent (REM) [5]. Further experiments show that the ambient and core body temperatures in humans affect the length and number of REM bouts [28]. As the ambient temperature deviates from thermoneutrality (around $29^{\circ} \mathrm{C}$ ), increasing effort needs to be exerted to thermoregulate, possibly requiring REM bouts to be interrupted in order to restore body temperature $[18,20]$. If the deviation is large enough, sleep may be interrupted or prevented all together. On the other hand, if the ambient temperature is thermoneutral, deep sleep is achieved faster and the number of REM cycles remains consistent while REM bouts elongate.

While mathematical models of human sleep regulation (reviewed in [6]) typically initiate the sleep period during the falling phase of the CBT as observed experimentally, and the model of Rempe et al. [39] addressed the circadian propensity for REM [12], very few mathematical models of sleep regulation have addressed temperature or thermoregulation. A notable exception is the collaboration of McGinty, Nakao, Szymusiak and 
colleagues. In a series of papers (see for example [31, 32, 33, 34]), they develop a mathematical model based on the hypothesis that homeostatic regulation of sleep is a form of long-term thermoregulation. They are motivated by data showing that manipulations that raise body temperature during the day may facilitate nighttime sleep (reviewed in [17]), suggesting a memory of heat load that dissipates during sleep. As their focus is on the homeostatic regulation of sleep, their models consider wake and NREM sleep, but not REM. In their models, NREM sleep propensity can be increased by current temperature effects (e.g., hypothalamic warming) or by memory of heat load earlier in the day. Quanten et al. [38] provide another example: they used a system identification approach to identify and model a feedback connection between distal heat loss and sleepiness. The data considered for their model did not include the occurrence of sleep, so the model addresses the onset of sleep but not sleep regulation.

In this paper we present a mathematical model of the sleep/wake cycle including REM and NREM sleep, and we use this model to study the effects of ambient temperature and thermoregulation on human sleep patterns. In Section 2, a description of the model is given, based on the model of Kumar et al. [24]. In Sections 3.1 and 3.2 we first separately analyze the sleep/wake and then REM/NREM circuits of the model without the effects of thermoregulation (i.e. at a thermoneutral state). We analyze the model with thermoregulation in Section 3.3, then compare our model results with experimental data in Section 3.4. Our results show that incorporating a fairly simple form of thermoregulation in a mathematical model for human sleep/wake cycling can account for several features in data from experiments on the effects of temperature on sleep. Our analysis provides a detailed understanding of the mathematical mechanisms and suggests that temperature effects could provide an effective constraint on mathematical models of human sleep/wake cycling.

\section{Mathematical Model.}

2.1. Neuron Populations Involved in Sleep/Wake Processes. Experimental investigations continue to identify areas of the brain responsible for initiating and maintaining states of the sleep cycle [8, 14] (see Fig. 1). Some key areas that are active during the waking state are the midbrain reticular formation (MRF) that helps maintain wakefulness by inhibiting sleep-active neurons, and the orexinergic neurons (ORX) of the hypothalamus that help stabilize wakefulness (see Fig. 2A). Damage to the ORX neurons can produce narcolepsy and hypersomnolence [8]. Neurons in the preoptic area of the hypothalamus (POAH) and in a population located caudally in the brainstem reticular formation (CRF) are sleep-active (see Fig. 2B) and have mutually-inhibitory connections with MRF neurons. Neurons that inhibit REM sleep (REM-off) can be found in several nuclei including the locus coeruleus, while neuron populations promoting REM sleep (REM-on) can be found in the laterodorsal/ pedunculopontine 


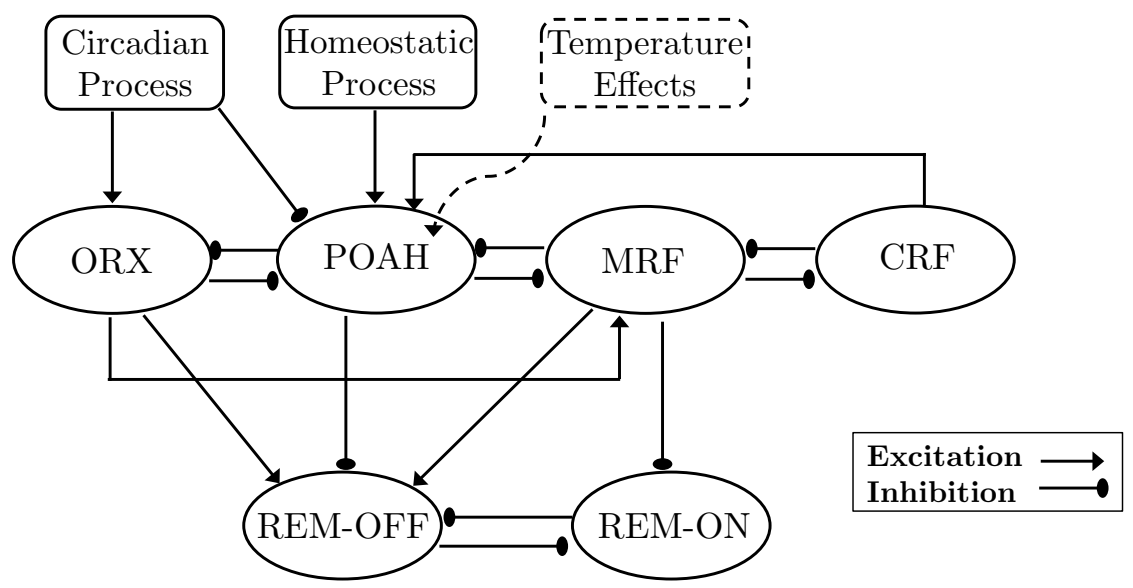

FIG. 1. Model diagram depicting the interactions between the neural populations (ovals) and processes (rounded rectangles) involved in the sleep-wake process considered by our model. Interactions between the populations are denoted by lines and can be excitatory (ending in an arrow) or inhibitory (ending in an oval).

tegmentum [36]. REM-on neurons are inhibited by MRF [8, 24, 27]. The suprachiasmatic nucleus (SCN) of the hypothalamus acts as an endogenous oscillator generating the circadian rhythm; it can be entrained to a 24-h light/dark cycle by inputs from the retina. The SCN excites the ORX neurons [37]. Many other neuronal populations have activity levels that vary with sleep/wake state and may participate in sleep/wake regulation. In this very brief overview we have focused on populations that will be explicitly included in the mathematical model presented below, as shown in Fig. 1 and Fig. 2. The model is based upon the model of Kumar et al. [24]; further details concerning the choice of neuronal populations to include and the construction of the mathematical model may be found there.

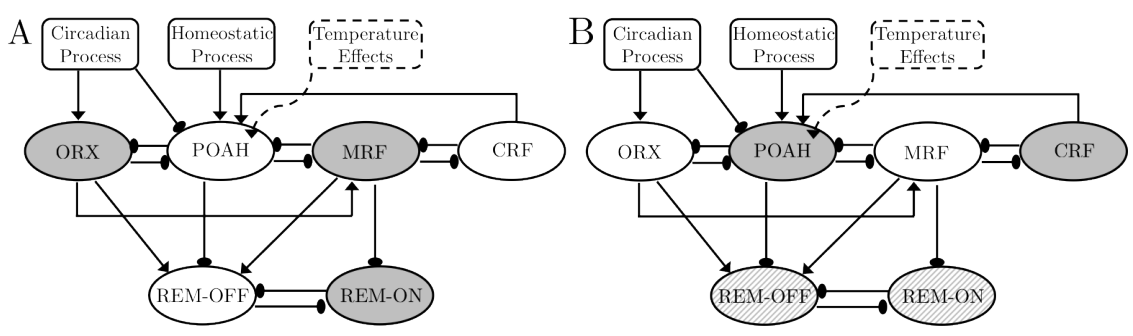

FIG. 2. Shaded populations indicate an active state during (A) Wake and (B) Sleep. Partly shaded regions indicate periods of alternating activity and inactivity.

2.2. Neuron Population Model. Our model contains six neuronal populations with directed excitatory and inhibitory connections as shown 
by the diagram in Fig. 1. Following [24], the mean activity level of each neuron population is modeled using Morris-Lecar (ML) equations [29]:

$$
\begin{aligned}
v_{i}^{\prime} & =I_{C a}\left(v_{i}\right)+I_{K}\left(v_{i}, w_{i}\right)+I_{l e a k}\left(v_{i}\right)+I_{i}-\sum I_{s y n} \\
& =g_{c a} m_{\infty}\left(v_{i}\right)\left(v_{c a}-v_{i}\right)+g_{k} w_{i}\left(v_{k}-v_{i}\right)+g_{l}\left(v_{l}-v_{i}\right)+I_{i}-\sum I_{s y n} \\
w_{i}^{\prime} & =\lambda_{\infty}\left(v_{i}, v_{3, i}, \phi_{i}, \tau_{i}\right)\left(w_{\infty}\left(v_{i}, v_{3, i}\right)-w_{i}\right),
\end{aligned}
$$

with $i=\{P O A H, C R F, O R X, M R F, R o n, \operatorname{Rof} f\}$. Notice that MorrisLecar model was originally developed to describe the excitable behavior of a single neuron. Here, with appropriate scaling of variables and time constants, we interpret the model variable $v_{i}$ as population activity or firing rate of the $i^{t h}$ neuron population. The term $I_{i o n}:=I_{C a}+I_{K}+I_{l e a k}$ represents the intrinsic propulation dynamics. Specifically, $I_{C a}$ (inward $\mathrm{Ca}^{2+}$ current in the original ML model) represents the fast regenerative mechanism for cycling and episode activity, which activates instantaneously $\left(m=m_{\infty}\left(v_{i}\right)\right) ; I_{K}$ (outward $\mathrm{K}^{+}$current in the original ML model) represents the slow negative feedback mechanism that terminates the activity episodes, governed by the activation variable $w_{i}$, here interpreted as the recovery variable; and, $I_{\text {leak }}$ represents the leak term involved in maintaining the resting state. The parameters $g_{l}, g_{c a}$ and $g_{k}$ are the maximum conductance values of the leak, $\mathrm{Ca}^{2+}$, and $\mathrm{K}^{+}$currents and $v_{l}, v_{c a}$ and $v_{k}$ are the associated equilibrium potentials. Steady state activation functions $m_{\infty}$ and $w_{\infty}$ are sigmoidal functions of the form:

$$
\begin{aligned}
m_{\infty}(v) & =0.5\left(1+\tanh \left(\frac{v-v_{1}}{v_{2}}\right)\right), \\
w_{\infty}\left(v, v_{3}\right) & =0.5\left(1+\tanh \left(\frac{v-v_{3}}{v_{4}}\right)\right) .
\end{aligned}
$$

The time constant $1 / \lambda_{\infty}$ for the recovery variable $w_{i}$ is voltage-dependent of the form:

$$
\lambda_{\infty}\left(v, v_{3}, \phi, \tau\right)=\frac{\phi}{\tau} \cosh \left(\frac{v-v_{3}}{2 v_{4}}\right) .
$$

Synaptic currents, $I_{\text {syn }}$ from neuron population $i$ to population $j$ have the general form

$$
I_{\text {syn }}=g_{s y n} s_{\infty}\left(v_{i}, v_{5, i}, v_{6, i}\right)\left[v_{j}-E_{s y n}\right],
$$

where $g_{s y n}$ is the strength of the $i j$ synapse, $E_{\text {syn }}$ is the synaptic reversal potential and $s_{\infty}$ is the activation function given by:

$$
s_{\infty}\left(v, v_{5}, v_{6}\right)=0.5\left(1+\tanh \left(\frac{v-v_{5}}{v_{6}}\right)\right) .
$$


Parameter values can be found in Table 2. For all inhibitory synaptic connections, $E_{\text {syn }}=-0.7$. Further, $\sum I_{\text {syn }}$ for each population involves a specific combination of currents with the following forms:

$$
\begin{aligned}
P O A H & : I_{M R F \rightarrow P O A H}+I_{C R F \rightarrow P O A H}+I_{O R X \rightarrow P O A H}+I_{\text {Tdev }}+I_{c i r}-I_{\text {hom }} \\
C R F & : I_{M R F \rightarrow C R F} \\
O R X & : I_{P O A H \rightarrow O R X}-I_{c i r} \\
M R F & : I_{P O A H \rightarrow M R F}+I_{C R F \rightarrow M R F}+I_{O R X \rightarrow M R F} \\
R E M_{o n} & : I_{M R F \rightarrow R o n}+I_{R o f f \rightarrow R o n} \\
R E M_{o f f} & : I_{M R F \rightarrow R o f f}+I_{O R X \rightarrow R o f f}+I_{\text {Ron } \rightarrow \text { Roff }}+I_{P O A H \rightarrow R o f f} .
\end{aligned}
$$

Further details about Morris-Lecar model parameters can be found in [29]. The evolution of variables $v_{P O A H}, v_{M R F}, v_{R o n}$ and $v_{R o f f}$ according to the equations above is shown in Fig. 3. Later, in Sections 3.1 and 3.2 we will provide a more detailed description of these dynamics.

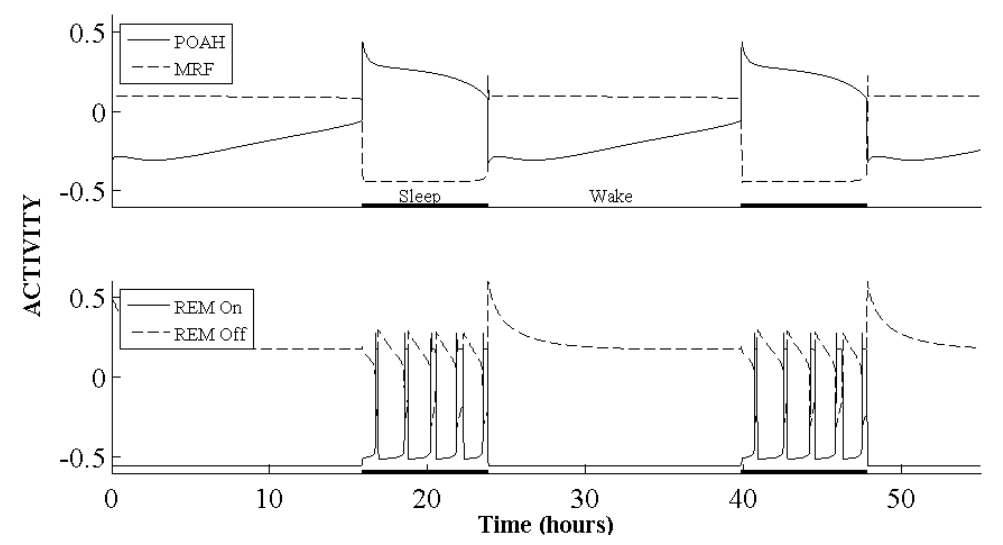

FIG. 3. The activity of various neuron groups during sleep wake cycling. Periods of sleep are indicated by bold horizontal lines along the $x$-axis.

2.3. Circadian Rhythm and Sleep Homeostatic Process. There are two forms of modulation of the sleep/wake system in our model at thermoneutrality. The SCN provides input to the POAH and ORX populations; we model this input as a circadian pacemaker, $C(t)$, as in [1]:

$$
\begin{aligned}
C(t)= & 0.97 \sin (\omega t)+0.22 \sin (2 \omega t)+0.07 \sin (3 \omega t) \\
& +0.03 \sin (4 \omega t)+0.01 \sin (5 \omega t)
\end{aligned}
$$

with $\omega=\frac{2 \pi}{24}$. The associated current is

$$
I_{\text {cir }}(t)=g_{\text {cir }}[C(t)+1]
$$


with synaptic coupling strength $g_{c i r}=0.3$. Since this current represents synaptic information from the SCN to other neuron populations, we have added a constant to $C(t)$ ensuring that the synaptic current between these neurons does not change sign. The inhibitory projection to POAH expresses a multi-synaptic inhibitory effect of SCN on sleep-promoting areas of the hypothalamus [39].

The second modulating process is the homeostatic drive for sleep described in $[1,11]$, which increases while awake and decreases during sleep. The homeostat variable $h$ is modeled by the equation

$$
h^{\prime}=\frac{(1-h)}{\tau_{1}} \mathcal{H}\left(v_{M R F}-v_{t h 2}\right)-\frac{h}{\tau_{2}} \mathcal{H}\left(v_{t h 2}-v_{M R F}\right),
$$

where $\mathcal{H}(\cdot)$ is the Heaviside step function. Notice that $h$ is dependent on the activity of the MRF. During wake, the MRF activity is above the threshold $v_{t h 2}$, the homeostat grows and thus the propensity toward sleep increases over time. Once asleep, with MRF activity below threshold, $h$ decreases. The associated current is

$$
I_{h o m}(h)=g_{h} h,
$$

which is excitatory to the POAH. Fig. 4 shows the time course of $I_{c i r}$ and $I_{\text {hom }}$ over several sleep/wake periods.

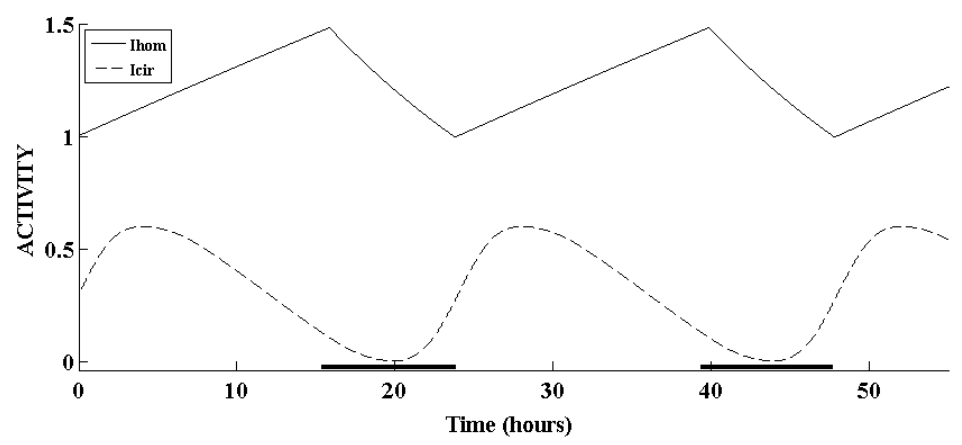

FIG. 4. Circadian and homeostatic currents.

2.4. REM Homeostat. The synaptic current from POAH to REMoff neurons, $I_{P O A H \rightarrow R o f f}$, includes an additional multiplicative term, syn, to incorporate a regulatory homeostat for REM sleep during the sleep phase. Specifically, this synaptic term takes the form

$$
\begin{aligned}
I_{P O A H \rightarrow R o f f}= & g_{P O A H \rightarrow R o f f} s_{\infty}\left(v_{P O A H}, v_{5, P O A H}, v_{6, P O A H}\right) \\
& \times\left[v_{R o f f}-E_{P O A H \rightarrow R o f f}\right] s y n,
\end{aligned}
$$


where syn is a dynamic variable that satisfies

$$
\begin{aligned}
\text { syn }^{\prime}= & \frac{(p-s y n)}{\tau_{3}} \mathcal{H}\left(v_{P O A H}-v_{t h}\right)-\frac{s y n}{\tau_{4}} \mathcal{H}\left(v_{t h}-v_{P O A H}\right) \\
p^{\prime}= & \frac{(1-p)}{\tau_{5}} \mathcal{H}\left(v_{\text {Roff }}-v_{t h}\right) \mathcal{H}\left(v_{P O A H}-v_{t h}\right) \\
& -\frac{p}{\tau_{6}}\left[\mathcal{H}\left(v_{t h}-v_{P O A H}\right)+\mathcal{H}\left(v_{R o n}-v_{t h}\right)\right] .
\end{aligned}
$$

This REM homeostat models the body's increased propensity for REM sleep while in NREM sleep and a decreased propensity for REM sleep while awake and in REM. During wake, when the activity of the POAH is low, and below threshold, $p$ decays to 0 at the rate of $1 / \tau_{6}$. During a REM bout, $p$ also decays and in NREM sleep, $p$ increases. Thus, during sleep, syn is generally attracted to $p$ and will grow toward the value of $p$ and decay to zero when wake is initiated; see Fig. 5 . We note that this REM homeostat differs from the implementation in the model of Kumar et al. [24]. Indeed, our REM homeostat also decays during REM and it is built on the inhibitory projection from the sleep-promoting area of the hypothalamus (POAH) to REM-off neurons. This connection is consistent with experiments [26] and has been included in other mathematical models of sleep regulation [39].

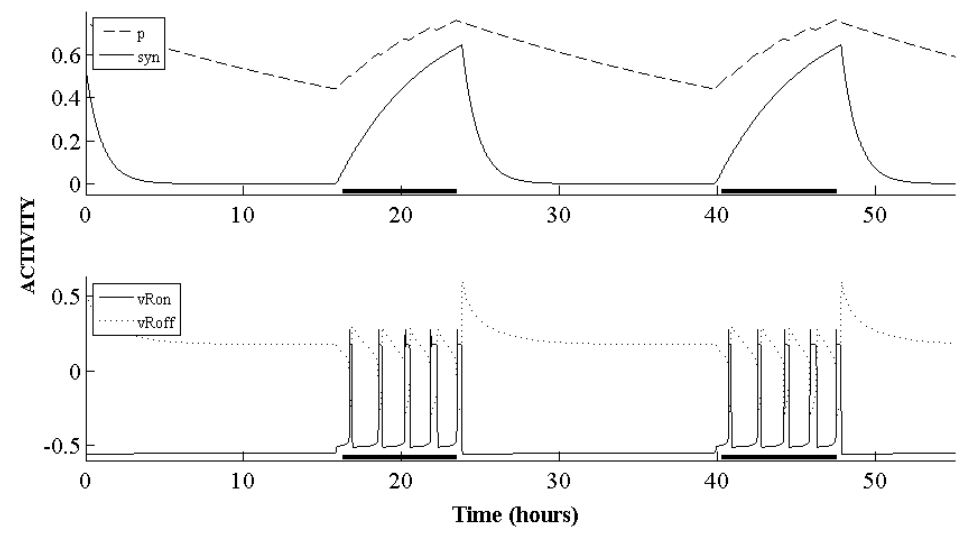

FIG. 5. REM sleep homeostat involved in the synaptic current from POAH to REM-off neurons (top) with REM-on and REM-off activity (bottom). 
2.5. Thermoregulation. Following [35], we model thermoregulation as

$$
\begin{aligned}
\tau_{v} T_{b}^{\prime}= & k\left(T_{a}-T_{b}\right)+T_{m}-\mathcal{H}\left(T_{b}-T_{s e t}\right) \frac{v_{c}\left(T_{b}-T_{s e t}\right)}{k_{c}+\left(T_{b}-T_{s e t}\right)} \\
& +\mathcal{H}\left(T_{\text {set }}-T_{b}\right) \frac{v_{h}\left(T_{\text {set }}-T_{b}\right)}{k_{h}+\left(T_{\text {set }}-T_{b}\right)}
\end{aligned}
$$

where $T_{b}$ is the core body temperature, $T_{a}$ is the ambient temperature and $T_{\text {set }}$ is the temperature that the body is trying to maintain (in ${ }^{\circ} \mathrm{C}$ ). $T_{\text {set }}$ fluctuates a half degree celsius around $37^{\circ} \mathrm{C}$ according to the circadian rhythm, i.e.

$$
T_{\text {set }}(t)=0.5 C(t)+37 .
$$

The first term in Eqn. (2.13) is the rate at which the body heats or cools as a function of the difference between ambient and body temperatures [40]. The second term, $T_{m}$, is the rate of endogenous metabolic heat production. The third and fourth terms model the processes of active thermoregulation. The third term (fourth term) describes the rate (modeled by a MichaelisMenten equation) at which system cools down (heats up, respectively) body temperature when it deviates from $T_{\text {set }}$. Constants $k_{c}$ and $k_{h}$ control the gain rate and constants $v_{h}$ and $v_{c}$ control the maximum rate. Notice that metabolism works differently for cooling than for heating if $k_{c}$ and $v_{c}$ constants are different than $k_{h}$ and $v_{h}$. Fig. 6 (black curve) shows the steady state values of $T_{b}$ for Eqn. (2.13) (with $T_{\text {set }}=37^{\circ} \mathrm{C}$ ) as a function of the ambient temperature $T_{a}$. Parameters controlling the metabolic processes responsible for thermoregulation were tuned in order to mimic biologically plausible ranges of environmental temperatures over which body temperature can be maintained $\left(10-37^{\circ} \mathrm{C}\right)$. Fig. 6 (grey curve) shows the steady state value of (2.13) when the metabolic process is shut down (during REM sleep) and the system does not thermoregulate. During wake and sleep humans thermoregulate with varying levels of efficiency; thermoregulating most efficiently during wake and less so during sleep [25]. The time constant $\tau_{v}$ controls the rate at which $T_{b}$ approaches the steady state and so we use different $\tau_{v}$ values during wake, $\tau_{v}=1$, and sleep (NREM and $\mathrm{REM}), \tau_{v}=500$.

2.5.1. Temperature Effects to POAH. There are temperature sensitive neurons in the POAH [23], and so our model incorporates the effects of ambient temperature through the activity of the POAH. During REM bouts, body temperature deviates from the set temperature. When this deviation exceeds a threshold of $0.2^{\circ} \mathrm{C}\left(T_{b}=T_{\text {set }}(t) \pm 0.2\right)$, then an inhibitory pulse is sent to the POAH in order to induce a brief awakening and restore thermoregulation (see Section 3.3 for an illustration of this mechanism). 


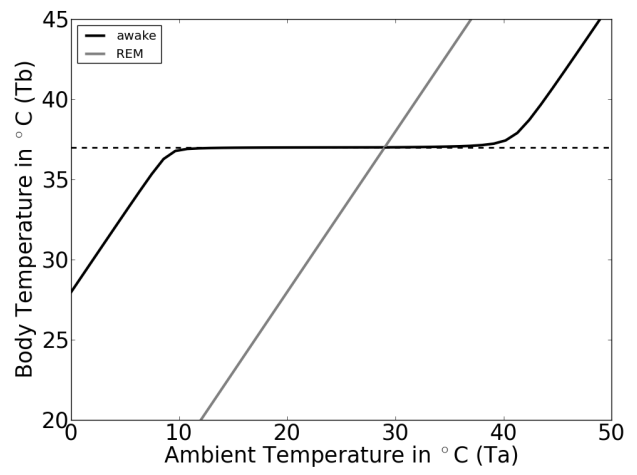

FIG. 6. Steady state value of thermoregulation Eqn. (2.13) as a function of ambient temperature $T_{a}$, with $T_{\text {set }}=37^{\circ} C, k_{h}=0.01, k_{c}=0.05, v_{c}=12$ and $v_{h}=20$ (black curve), and steady state value of Eqn. (2.13) when the metabolic processes are not functioning, $v_{c}=0$ and $v_{h}=0$ (grey curve). Dashed curve indicates thermoneutrality $T_{b}=37^{\circ} \mathrm{C}$.

The inhibitory current $I_{\text {Tdev }}$ is modeled as the product of a conductance with a voltage difference according to the following equations:

$$
I_{T d e v}=g_{T d e v} s_{T d e v}\left(v_{P O A H}-E_{s y n}\right),
$$

where $g_{T \text { dev }}=1.5$ is the maximal synaptic conductance and $s_{T d e v}$ depends on the temperature in the following way:

$$
s_{\text {Tdev }}^{\prime}=I_{\text {pulse }}\left(1-s_{\text {Tdev }}\right) / \tau_{r}-s_{\text {Tdev }} / \tau_{d},
$$

with $\tau_{r}=0.1, \tau_{d}=2 . I_{\text {pulse }}$ jumps to 1 whenever body temperature $T_{b}$ falls below a certain minimum $T_{\min }=T_{\text {set }}-0.2$ or reaches above a certain maximum $T_{\max }=T_{\text {set }}+0.2$, i.e.

$$
I_{\text {pulse }}=\mathcal{H}\left(T_{\text {min }}-T_{b}\right)+\mathcal{H}\left(T_{b}-T_{\text {max }}\right) .
$$

Thus $s_{\text {Tdev }}$ rises while $I_{\text {pulse }}=1$ and decays to 0 otherwise.

\section{Results.}

3.1. Sleep/Wake Circuit. Following Rempe et al [39] and Kumar et al [24], we assume the inputs a population receives evolve on a much slower time scale than the dynamics of $v_{i}, w_{i}$ dictated by Eqn. (2.1). With this fast-slow decomposition, we can understand the maintenance of wake and sleep states, as well as the transitions between them, by the solutions of Eqn. (2.1) governed by the nullclines of the $\left(v_{i}, w_{i}\right)$ phase plane (see also [7]). We provide a description of the sleep/wake cycle (see Fig. 3 top) during thermoneutrality based on the evolution of the nullclines on the $\left(v_{P O A H}, w_{P O A H}\right)$ and $\left(v_{M R F}, w_{M R F}\right)$ phase planes. 
TABLE 1

Parameter values for each neuron population. ${ }^{*} \tau_{R E M o f f}$ is described by a function, Eqn. (3.1), dependent on the potential of the REM-off neurons, rather than a constant value.

\begin{tabular}{|c|c|c|c|c|c|}
\hline \multirow{2}{*}{ neuron population } & \multicolumn{5}{|c|}{ parameter values } \\
\cline { 2 - 6 } & $v_{3, i}$ & $v_{5, i}$ & $\phi_{i}$ & $I_{i}$ & $\tau_{i}$ \\
\hline$P O A H$ & -0.15 & 0 & 0.01 & 0 & 1 \\
$C R F$ & -0.15 & -0.1 & 0.05 & 0.7 & 1 \\
$O R X$ & 0.1 & -0.1 & 1 & 0 & 1 \\
$M R F$ & 0.1 & -0.1 & 1 & 0 & 1 \\
$R E M_{\text {on }}$ & 0.18 & 0 & 0.1 & 0 & 0.05 \\
$R E M_{\text {off }}$ & 0 & 0 & 0.1 & 0.7 & $*$ \\
\hline
\end{tabular}

TABLE 2

Parameters used in the model for neuron populations

\begin{tabular}{|c|c|c|c|c|c|}
\hline parameter & value & parameter & value & parameter & value \\
\hline$g_{C R F \rightarrow P O A H}$ & 0.5 & $g_{M R F \rightarrow R o n}$ & 0.5 & $v_{c a}$ & 1 \\
$g_{M R F \rightarrow P O A H}$ & 0.5 & $E_{O R X \rightarrow R o f f}$ & 0 & $v_{1}$ & -0.01 \\
$g_{O R X \rightarrow P O A H}$ & 1 & $E_{O R X \rightarrow M R F}$ & 0 & $v_{2}$ & 0.15 \\
$g_{P O A H \rightarrow M R F}$ & 0.5 & $E_{C R F \rightarrow P O A H}$ & 0 & $v_{4}$ & 0.145 \\
$g_{C R F \rightarrow M R F}$ & 0.2 & $E_{M R F \rightarrow R o f f}$ & 0.4 & $v_{6}$ & 0.1 \\
$g_{O R X \rightarrow M R F}$ & 1 & $\tau_{3}$ & 300 & $v_{6, P O A H}$ & 0.01 \\
$g_{M R F \rightarrow C R F}$ & 1 & $\tau_{4}$ & 100 & $v_{7}$ & 0.01 \\
$g_{P O A H \rightarrow O R X}$ & 1 & $\tau_{5}$ & 700 & $g_{l}$ & 0.5 \\
$g_{R o n \rightarrow R o f f}$ & 0.2 & $\tau_{6}$ & 3000 & $g_{k}$ & 2 \\
$g_{P O A H \rightarrow R o f f}$ & 1.6 & $v_{t h}$ & 0 & $g_{c a}$ & 1.33 \\
$g_{M R F \rightarrow R o f f}$ & 0.5 & $v_{t h 2}$ & -0.1 & $g_{c i r}$ & 0.3 \\
$g_{O R X \rightarrow R o f f}$ & 0.5 & $v_{l}$ & -0.1 & $g_{h}$ & 7 \\
$g_{R o f f \rightarrow R o n}$ & 1.1 & $v_{k}$ & -0.7 & & \\
\hline
\end{tabular}

Considering the nullclines of Eqn. (2.1) for frozen synaptic input, we have a cubic shaped $v$-nullcline $\left(v^{\prime}=0\right)$ and a sigmoidal shaped $w$-nullcline $\left(w^{\prime}=0\right)$. See Fig. 7B and C. Each neuronal population receives input from other populations. As the amount of input received changes over time, the $v$-nullcline changes its position on the phase plane and crosses the $w$ nullcline at different points corresponding to different states. Notice that the dynamics on the phase plane is also of the slow-fast type-dynamics of $v$ is faster than $w$. When $v$-nullclines are high (indicating strong excitatory or low inhibitory drive), they cross the $w$-nullcline on the right branch defining a "stable fixed point" of high activity (notice that this is a real fixed point only when the synaptic input is frozen). On the contrary, when $v$ nullclines sit low (indicating strong inhibitory or low excitatory drive), they cross the $w$-nullcline on the left branch defining a "stable fixed point" of low activity. Finally, when $v$-nullclines cross the $w$-nullcline on the middle 
branch, they define an "unstable fixed point" and a "stable limit cycle" of periodic activity and inactivity naturally occurs (see [7]).

Thus, at the beginning of the wake state (see red dot in Fig. 7A), the

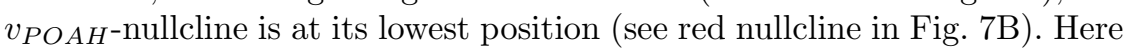
the excitatory drive from the sleep homeostat is at its minimum value and the inhibitory input from the circadian rhythm is rising (see Fig. 4). The system sits on the "stable fixed point" (intersection of $v_{P O A H}$ and $w_{P O A H^{-}}$ nullclines) that lies on the left branch of the $v_{P O A H}$-nullcline (silent state). At this moment, MRF is not receiving inhibition from POAH (silent) and the $v_{M R F}$ and $w_{M R F^{-}}$nullclines intersect on the right branch of the $v_{M R F^{-}}$ nullcline (active state), see red nullcline in Fig. 7C. During the wake state the homeostat rises (see Fig. 4) and the $v_{P O A H}$-nullcline moves upward. The trajectory is then tracking the "stable fixed point" located on the left branch until it transitions to the middle branch and destabilizes ( $v_{P O A H^{-}}$ nullcline transitions from red to green nullcline in Fig. 7B). At this point, the trajectory jumps up to the right branch pushed by the fast dynamics of Eqn. (2.1), indicating the onset of the sleep period (see green dot in Fig. 7A). Since POAH inhibits MRF, the $v_{M R F}$-nullcline moves downward (blue nullcline in Fig. $7 \mathrm{C}$ ) and $v_{M R F}$ approaches the new fixed point corresponding to a silent state. Now, POAH is not receiving inhibition from MRF (or ORX) anymore and the $v_{P O A H}$-nullcline moves further up (blue nullcline in Fig. 7B). During the sleep period, the homeostatic drive is reduced while the circadian inhibitory input to POAH rises (see Fig. 4), causing a downward shift to $v_{P O A H}$-nullcline $\left(v_{P O A H}\right.$-nullcline transitions from blue to orange nullcline in Fig. 7B). The sleep period terminates when the $v_{P O A H}$-nullcline shifts sufficiently low such that the "stable fixed point" moves to its middle branch and destabilizes (orange nullcline in Fig. 7B) and fast dynamics pushes the trajectory back down to the left branch. Immediately after that, MRF is released from POAH inhibition, $v_{M R F}$-nullcline moves back up (from orange to cyan nullcline in Fig. $7 \mathrm{C}$ ) and MRF activates again, which in turn suppresses POAH further more

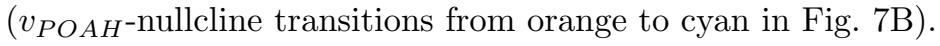

3.2. The REM Subcircuit. The REM subcircuit consists of a flipflop circuit between the REM-on and REM-off neuronal groups. The description below shows that the REM-off population is driving this subcircuit and the REM-on population is passive. During sleep both the MRF and ORX neuronal groups are suppressed and the POAH is active (Fig. 2B). Thus, in order to analyze the regulation of REM cycling during sleep we isolate the REM subcircuit by ignoring the connections from the MRF and the ORX to the REM subcircuit and making the strength of the inhibitory input from the POAH governed by the REM homeostatic process, syn, a parameter (see Eqn. (2.12)). Notice that the equation for $w_{R o f f}$ in (2.1) does not depend on the REM homeostatic process. Thus, as we simulate the rise of the REM homeostat, the sigmoidal shaped graph of the 


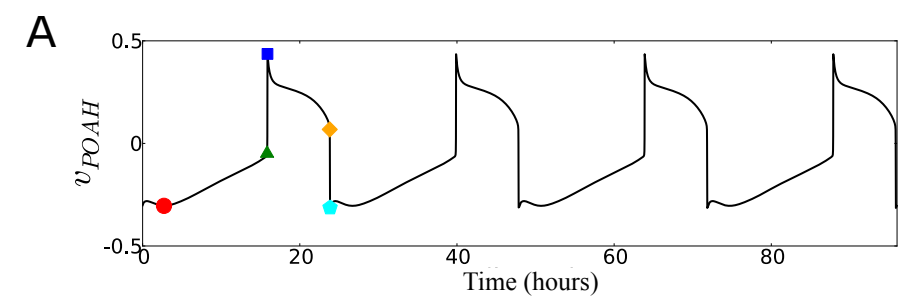

B

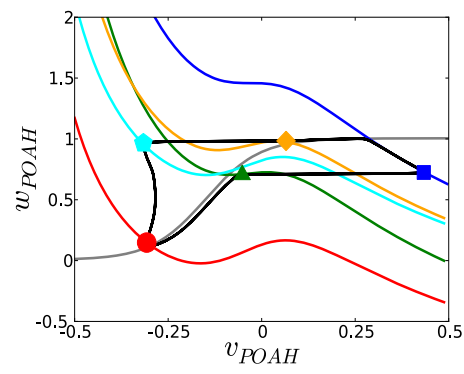

C

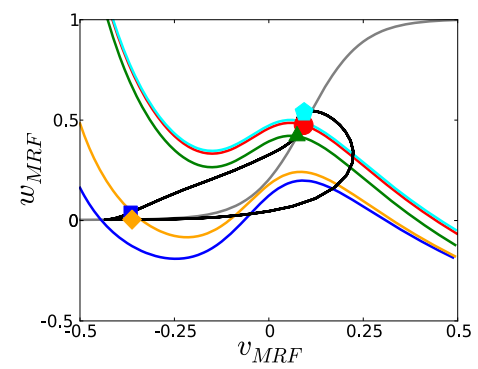

FIG. 7. (A) Time course of $v_{P O A H}$ over four cycles for thermoneutrality. (B) Projection of the trajectory onto the $\left(v_{P O A H}, w_{P O A H}\right)$-phase plane (black curve), together with the $w_{P O A H}$-nullcline (grey curve) and the position of the $v_{P O A H}$-nullcline at five different times indicated with symbols in panel $A(C)$ Projection of the trajectory onto the $\left(v_{M R F}, w_{M R F}\right)$-phase plane (black curve), together with the $w_{M R F}$-nullcline (grey curve) and the position of the $v_{M R F}$-nullcline at five different times indicated with symbols in panel $A$ (color of $v$-nullcline in $B$ and $C$ matches symbol color in panel A).

$w_{R o f f}$-nullcline remains unchanged and the $v_{R o f f}$-nullcline makes a vertical shift downward, changing the dynamics of the subsystem. In Fig. 8A, we show the bifurcation diagram of the REM subsystem as syn (now a parameter) is varied. We see that for small values of syn the system has a stable equilibrium point with high $v_{R o f f}$, which loses stability through a Hopf bifurcation (for syn $=0.327$ ), where a stable limit cycle is born. The stable limit cycle persists over a range of syn values $(\operatorname{syn} \in(0.327,0.668))$ and disappears via another Hopf bifurcation (for syn =0.668). In Fig. 8B we show the period of the oscillations. After the second Hopf bifurcation, the system has a stable equilibrium point with low $v_{R o f f}$. Notice that for a range of syn values the system presents three equilibrium points that are born or disappear through saddle node bifurcations, and only one or none are stable for a given syn. The following is a discussion of these dynamical regimes in more detail based on the position of the nullclines.

For lower values of the REM homeostat (small syn), like during sleep onset, REM-off neurons are active. Indeed, the $v_{R o f f}$-nullcline intersects

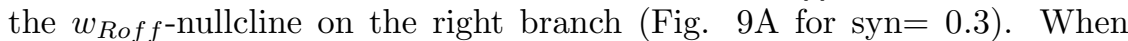
$v_{\text {Roff }}>v_{\text {th }}$ the inhibitory input to REM-on neurons force $v_{R o n}<v_{\text {th }}$, 

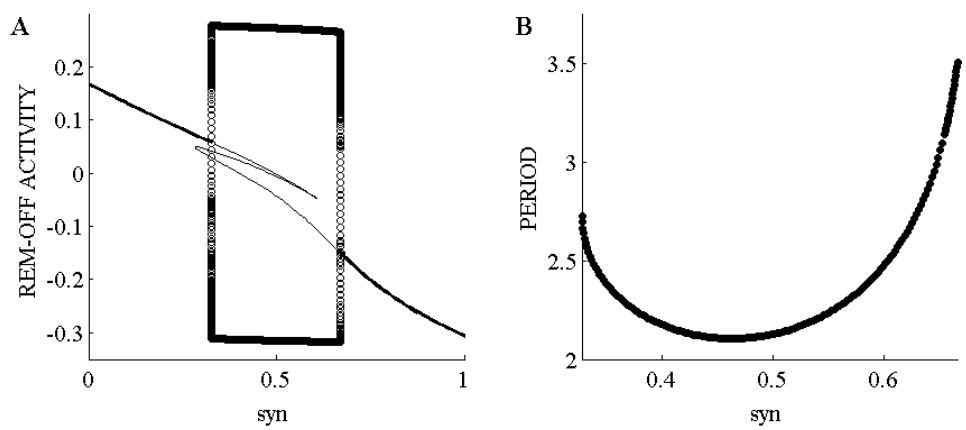

FIG. 8. (A) Bifurcation diagram of REM subsystem via REM-off activity as syn (here a parameter) is varied. Thick and thin lines are stable and unstable fixed orbits, respectively. Circles are periodic orbits (B) Period of the oscillations for the stable limit cycle versus syn. Diagrams were computed using xppaut.

thus suppressing REM-on. The $v_{R o n}$-nullcline intersects the $w_{R o n}$-nullcline on the left branch (Fig. 9B for syn =0.3). When the REM homeostat is at its maximum, the $v_{R o f f}$-nullcline shifts down and intersects the $w_{R o f f^{-}}$ nullcline on the left branch (Fig. 9A for syn $=0.7$ ). Thus REM-off neurons are inactive and there is no inhibitory input to REM-on neurons. Hence we see a stable fixed point on the right branch of the $v_{R o n}$-nullcline (Fig. 9B for $\operatorname{syn}=0.7)$. These system behaviors allow us to model two important typical characteristics of sleep in a healthy human adult: sleep begins with NREM sleep and ends in REM sleep.
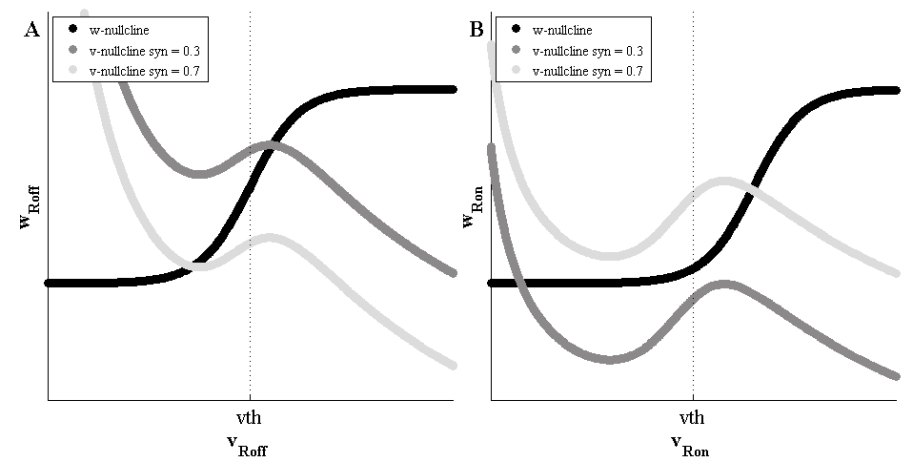

FIG. 9. Projection of the $v$ and w-nullclines on the (A) $\left(v_{R o f f}, w_{R o f f}\right)$-phase space and $(B)\left(v_{R o n}, w_{R o n}\right)$-phase space for values of syn for which the REM/NREM subsystem has a stable fixed point. Dark-grey v-nullclines correspond to a low value of the REM homeostat (syn $=0.3$ ); indicating that at the sleep onset REM-off is active and REM-on is suppressed. Similarly, light-grey v-nullclines correspond to high values of the REM homeostat (syn = 0.7); indicating that at the end of sleep REM-off is inactive and REM-on is active. 
In a different regime of the REM homeostat (see Fig. 8A for syn $\in$ $(0.327,0.668))$ the system has an attracting limit cycle (Fig. 10A). Notice that since the $v_{R o f f}$-nullcline depends also on the current value of $v_{R o n}$, during the active phase of $v_{R o f f}$ (silent phase, respectively) the trajectory on the REM-off phase plane is tracking the right branch (left branch) of the $v_{R o f f}$-nullcline corresponding to a low value (high value) of $v_{R o n}$. Fig. 10B shows the projection of the limit cycle onto the $\left(v_{R o n}, w_{R o n}\right)$ phase plane, where the REM-on population transitions between two "fixed points" (these are real fixed points only when $v_{R o f f}$ is frozen at its minimum and maximum values) as $v_{R o f f}$ ranges between its minimum and maximum values. We have made the parameter $\tau_{R o f f}$ a function of $v_{R o f f}$, so that in this state the REM-off trajectory travels up along the active phase (right branch of the $v_{R o f f}$-nullcline) slower than traveling down the silent phase (left branch of the $v_{R o f f}$-nullcline). Thus, we have:

$$
\tau_{\text {Roff }}\left(v_{\text {Roff }}\right)=\tau_{\text {low }}+\tau_{\text {high }} \mathcal{H}\left(v_{\text {Roff }}-v_{\text {th }}\right)
$$

with $\tau_{\text {low }}=5$ and $\tau_{\text {high }}=35$. This was done in the model so that for a given value of the REM homeostat the duration of a NREM bout is longer than a REM bout, which agrees with [25].

As the REM homeostat continues to increase (see Fig. 5), while in the oscillatory regime, we are able to see a lengthening of the REM/NREM periods during the night (Fig. 8B). Moreover, since the position of the $v_{R o f f}$-nullcline moves downward on the $\left(v_{R o f f}, w_{R o f f}\right)$-phase space as $s y n$ increases, it has the effect of lengthening the portion of the silent branch of the $v_{R o f f}$-nullcline that the trajectory is tracking while shortening the active branch. Therefore, we are also able to model an increase in the length of REM bouts as sleep progresses (see Fig. 3 bottom).

3.3. Effects of Thermoregulation on Sleep Dynamics. Whenever body temperature $T_{b}$, described by Eqn. (2.13), deviates more than $0.2^{\circ} \mathrm{C}$ from thermoneutrality (typically during REM sleep; see Fig. 11), it triggers an inhibitory pulse $I_{T d e v}$ to POAH, described by Eqn. (2.15). This produces the suppression of $\mathrm{POAH}$ activity, resulting in a brief awakening (see Fig. 11). Next, we discuss the dynamical effects of the inhibitory current $I_{\text {Tdev }}$ on the dynamics of POAH based on the nullclines approach. See Fig. 12 for $T=24^{\circ} \mathrm{C}$. During sleep, POAH is activated and the trajectory moves towards a fixed point (active state) located on the right branch of the $v_{P O A H}$-nullcline (see red curve and dot in Fig. 12B). An inhibitory pulse (due to deviation from thermoneutrality) shifts the $v_{P O A H}$-nullcline downward (green curve in Fig. 12B), the trajectory then lies above the right knee of the $v_{P O A H}$-nullcline (see green dot on the trajectory) and fast dynamics of Eqn (2.1) pushes the orbit towards the left branch of the $v_{P O A H^{-}}$ nullcline. Thus, the POAH population becomes silent (see Fig. 12A), releasing the MRF population, which activates. Since the duration of the inhibitory pulse is very short, soon after that, the amount of inhibition 

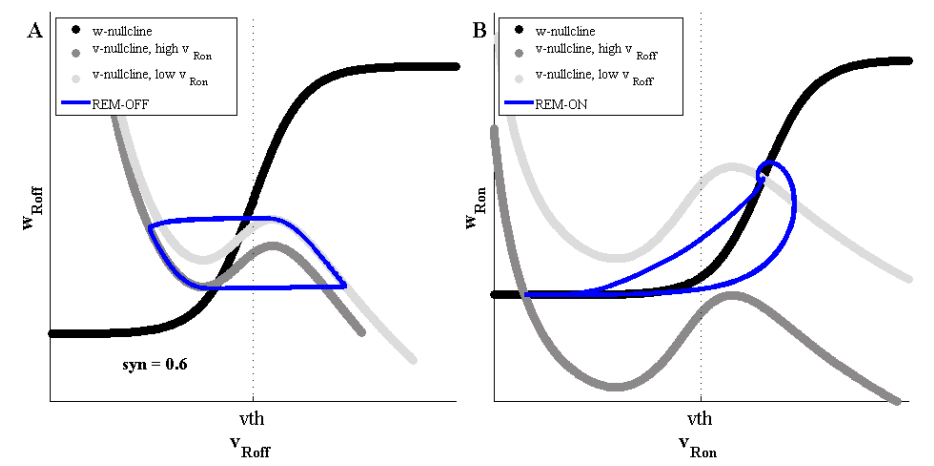

FIG. 10. Projection of the stable limit cycle for syn $=0.6$ (blue) together with the $v$-nullclines (grey) and the w-nullclines (black) onto the $(A)\left(v_{R o f f}, w_{R o f f}\right)$-phase space and $(B)\left(v_{R o n}, w_{R o n}\right)$-phase space. As this is a 4-dimensional system, the $v$ nullclines in a given phase-plane move up and down as we trace the trajectory in the other phase-plane.

that POAH receives is reduced. This has the effect, together with a weak inhibitory drive from MRF and ORX (see text below for more details) as well as high homeostatic drive and low inhibitory input from the circadian

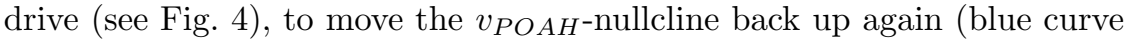
in Fig. 12B). Now, the trajectory lies below the left knee of the $v_{P O A H^{-}}$ nullcline (see blue dot on the trajectory and blue nullcline in Fig. 12B) and again fast dynamics pushes the orbit towards the right branch of the $v_{P O A H^{-n u l l c l i n e . ~ T h e ~ t r a j e c t o r y ~ t h e n ~ m o v e s ~ a l o n g ~ t h e ~ r i g h t ~ b r a n c h ~ t o-~}}$ wards the fixed point (again in the active state, see cyan and red dots in Fig. 12B). Thus, the POAH population activates again (see cyan dot on Fig. 12A), suppresses the MRF population and this terminates the brief awakening.

We would like to emphasize that we introduced some dynamics in the inhibitory connections from ORX and MRF to POAH (they are strong during wake but weakened during sleep) in order to simulate brief awakenings observed in experimental data. Consider, for instance, the case when an awakening ( $v_{P O A H}$ is suppressed due to an inhibitory pulse) is produced soon after falling sleep or at early stages of the night. Then, if the inhibitory strength from MRF and ORX to POAH is as during wake, the $v_{P O A H}$-nullcline would correspond to the green one in Fig. 7B. So, during the awakening period the trajectory would move along the left branch of the green nullcline in Fig. 7B until it would reach the left knee and jump up again, causing the system to fall back asleep. These dynamics would produce awakenings that are of the order of half an hour (results not shown). However, brief awakenings are of the order of a few minutes. We observed that by reducing the amount of inhibition POAH receives during brief awakenings, the $v_{P O A H}$-nullcline can be shifted further up, reducing 

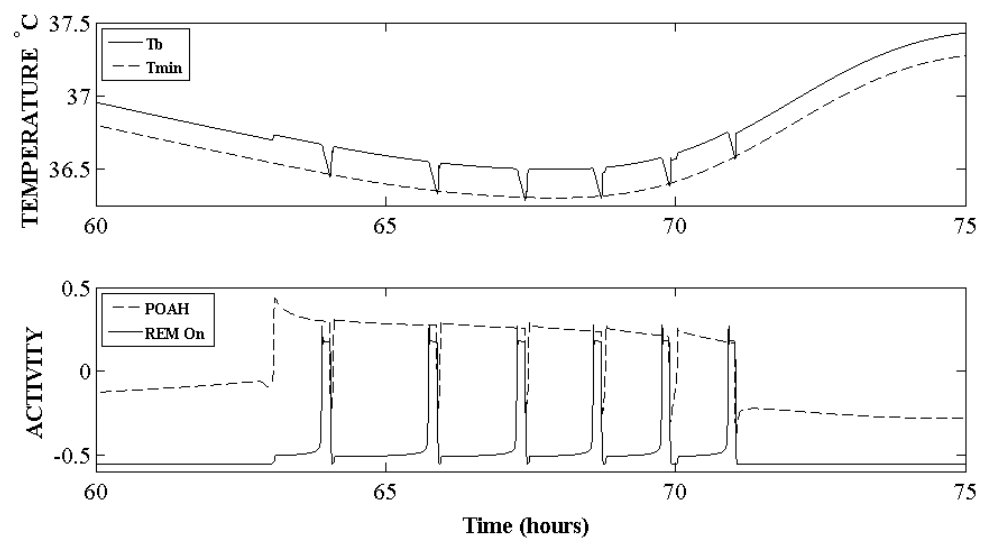

FIG. 11. Body temperature and the $\tau_{\text {min }}$ function (top) with the associated POAH and REM-on activity (bottom) during one night with an ambient temperature of $21^{\circ} \mathrm{C}$. During a REM cycle, when the body cannot thermoregulate, body temperature, $T_{b}$, will deviate away from thermoneutral until it reaches the minimum function value. At which time the body will leave REMS and wake so it can begin thermoregulating again.

the time the trajectory travels along the left branch before reaching the left knee (compare the position of the blue nullcline in Fig. 12B with the position of the green nullcline in Fig. 7B). We did so by modulating the strength of inhibition from MRF and ORX to POAH, so that it is strong during wake but it is weakened during sleep. Mathematically, we multiply Eqn. (2.5) for the synaptic currents $I_{O R X \rightarrow P O A H}$ and $I_{M R F \rightarrow P O A H}$ by a new variable $s y n_{p}$ that evolves according to the following differential equation:

$\operatorname{syn}_{p}^{\prime}=\left(0.2-\operatorname{syn}_{p}\right) \mathcal{H}\left(v_{P O A H}-v_{t h}\right) / \tau_{3 p}+\left(1-\operatorname{syn}_{p}\right) \mathcal{H}\left(v_{t} h-v_{P O A H}\right) / \tau_{4 p}$,

where $\tau_{3 p}=50$ and $\tau_{4 p}=100$. These dynamics do not affect the duration of the awake period during the day. Indeed, at the end of the sleep period the sleep homeostat is at its minimum level and the inhibitory input from the circadian drive to POAH is high, thus when an awakening is produced, the $v_{P O A H}$-nullcline is located a bit further down (see cyan nullcline in Fig. 7). Since more time is spent on the left branch, inhibitory synapses from MRF and ORX to POAH recover their strength and push the $v_{P O A H^{-}}$ nullcline even further down (cyan and red nullclines in Fig. 7), causing the long awake period during the day.

3.4. Comparison with Experimental Data. Fig. 13 shows the time course of $\mathrm{POAH}$ activation across a single night at the following ambient temperatures: $21^{\circ} \mathrm{C}, 24^{\circ} \mathrm{C}, 29^{\circ} \mathrm{C}, 34^{\circ} \mathrm{C}$ and $37^{\circ} \mathrm{C}$. In the case in which the ambient temperature is thermoneutral $\left(29^{\circ} \mathrm{C}\right)$, no awakenings occur during the night. Due to the increased need for thermoregulation, 
A

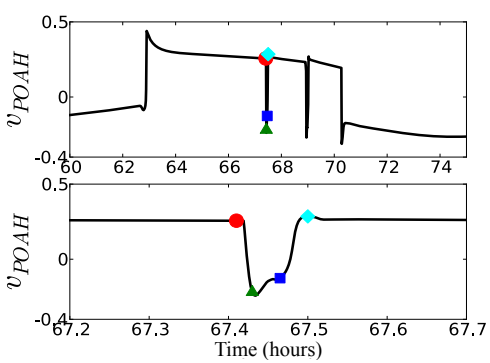

$\mathrm{B}$

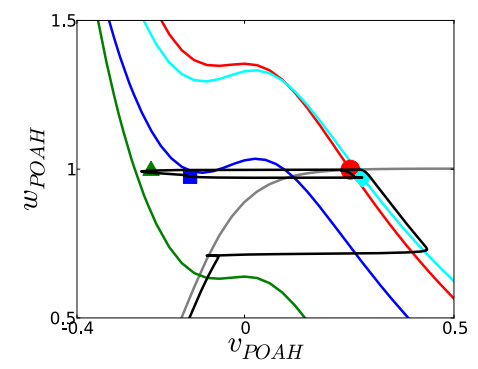

FIG. 12. (A) Time course of $v_{P O A H}$ during one night with ambient temperature $T_{a}=24^{\circ} \mathrm{C}$ (top) and zoom around the first brief awakening (bottom). (B) Projection of the trajectory in $A$ until the end of the first awakening onto the $\left(v_{P O A H}, w_{P O A H}\right)$-phase plane (black curve), together with the $w_{P O A H}$-nullcline (grey curve) and the position of the $v_{P O A H}$-nullcline at four different times around the first brief awakening indicated with symbols in panel $A$ (color of $v_{P O A H}$-nullcline in $B$ matches symbol color in panel A)

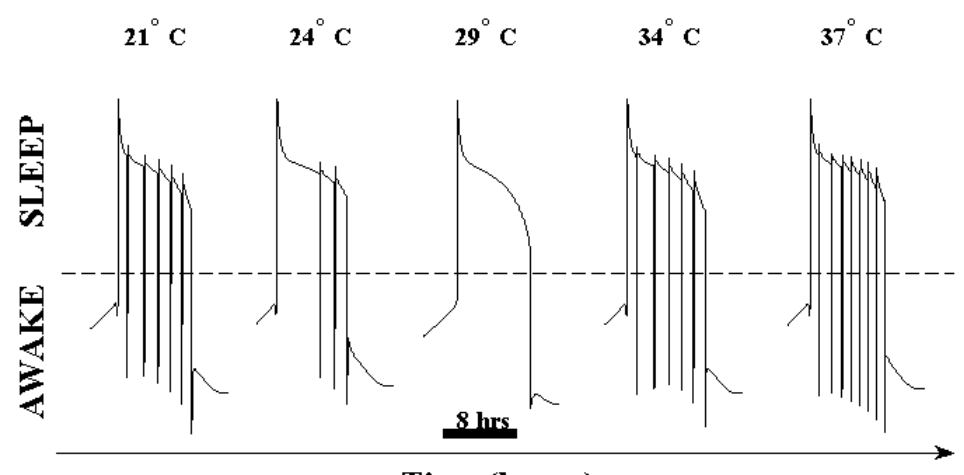

Time (hours)

FIG. 13. POAH activity during one night, including awakenings at various ambient temperature values.

the number of awakenings per night increases as the ambient temperature deviates further from thermoneutral, with a maximum of seven awakenings across a single night at ambient temperature $37^{\circ} \mathrm{C}$.

Further numerical results concerning the affects of ambient temperature on night awakenings is included in Table 3. Here, we see that, across all simulated ambient temperatures, the length of the night is $8 \pm 0.5$ hours. Our model displays a significant reduction in REM sleep at $37^{\circ} \mathrm{C}$ as opposed to other temperatures. This is consistent with the works of $[18,20]$ in which the authors found that sleeping at temperatures extremely above 
TABLE 3

Results of model simulations at various ambient temperatures.

\begin{tabular}{|c|c|c|c|c|c|}
\cline { 2 - 6 } \multicolumn{1}{c|}{} & \multicolumn{5}{c|}{ Temperature $\left({ }^{\circ} \mathrm{C}\right)$} \\
\cline { 2 - 6 } \multicolumn{1}{c|}{} & 21 & 24 & 29 & 34 & 37 \\
\hline Avg. Night Length (hr) & 7.96 & 7.56 & 7.96 & 8.41 & 8.00 \\
Num. Awakenings per night & 5 & 2 & 0 & 5 & 7 \\
Avg. Awakening Length (min) & 4.55 & 3.78 & $\mathrm{n} / \mathrm{a}$ & 4.52 & 4.71 \\
Num. REM Cycles & 6 & 5 & 5 & 6 & 8 \\
Mean REM Length (min) & 8.32 & 12.56 & 16.90 & 10.11 & 4.25 \\
\hline
\end{tabular}

thermoneutral leads to both increased amounts of wakefulness and a reduction of time spent in REM. Also consistent with the findings of [18], model simulations produce an increased amount of wakefulness observed at $21^{\circ} \mathrm{C}$ when compared to temperatures closer to thermoneutral. Our model predicts only small variations in the number of REM cycles across all temperatures except at $37^{\circ} \mathrm{C}$. The consistency in the number of REM cycles generally coincides with the experimental findings of Muzet et. al [30]. However, [30] found fewer REM bouts across the night (4 to 5) when compared to our model ( 5 to 8 ). This fact can be rectified by noting that Muzet et. al combined two REM bouts if they were separated by less than 15 minutes. This combined counting of REM episodes might account for the disparity between the data presented in [30] and the simulations of the current model.

4. Discussion. We have presented a model of human sleep/wake regulation with thermoregulation and temperature effects. At thermoneutrality, the model accounts for features of normal sleep in a circadian-entrained individual including the timing and duration of sleep, the number and duration of REM/NREM cycles, initiating sleep with NREM and waking from REM sleep. REM bouts lengthen across the first four REM/NREM cycles, but the fifth REM bout is terminated by wakefulness before reaching the duration of the fourth REM bout $[2,25]$. Thermoregulation is less efficient during NREM sleep compared to during wakefulness, modeled by increasing the time constant of the active thermoregulation process. During REM sleep, thermoregulation is suspended, and ambient temperatures away from thermoneutrality may result in excessive drift of the core body temperature (CBT). In the model, REM sleep is then interrupted in order to restore thermoregulation, and sleep does not resume until CBT has been corrected. Such brief awakenings have been observed in experiments in which subjects attempt sleep at a range of temperatures. In agreement with data, the model shows that the number and duration of the brief awakenings increase as temperatures either increase or decrease away from thermoneutrality. These interruptions result in shorter REM durations, so that REM bouts are longest at thermoneutrality and become shorter as 
the temperature moves away from thermoneutrality.

Czeisler et al. [9] report a particularly close relation between CBT and the rhythm of REM sleep propensity. Interestingly, the model shows trends for REM durations that vary with ambient temperature. At the coldest temperature modeled $\left(21^{\circ} \mathrm{C}\right), \mathrm{REM}$ durations are strictly decreasing across the night; they strictly increase at $34^{\circ} \mathrm{C}$. At $24^{\circ} \mathrm{C}$, they increase and then decrease, while at $37^{\circ} \mathrm{C}$ they decrease and then increase. The variation in trend is due to the nonlinear interaction between the ambient temperature, thermoregulation, and the circadian rhythm of CBT. At $24^{\circ} \mathrm{C}$, some model REM bouts were interrupted due to temperature deviations, while others were not. These results point out that, in order to understand the effect of ambient temperature on REM bout durations, it is important to record the circadian phase of each REM bout as well as its duration.

Our model is based on the model of Kumar et al. [24], but with several modifications in addition to thermoregulation. We did not include any nuclei or connections for which the model in [24] used a default synaptic conductance of 0 ; this did not affect the dynamics of the model under normal conditions. We added an inhibitory connection from the circadian process to POAH; such a connection has been included in other mathematical models [39] and summarizes a multisynaptic inhibitory influence. We moved the REM homeostat from the excitatory CRF to REM-on synapse (which we removed) to a new, inhibitory POAH to REM-off connection. These modifications ensured that the NREM/REM cycle at thermoneutrality had characteristics reported from experiments: 4-5 NREM/REM cycles per night, REM bouts lengthen across the night, and REM bouts are shorter than NREM bouts. While the biological mechanisms underlying the dynamics of REM/NREM cycles are not yet understood, it is interesting to note that in our model the REM-on neurons fire opportunistically while the REM-off population drives the cycle. In [24], the REM-on populations drive the NREM/REM cycles. Both models have a mutually inhibitory flip-flop circuit for NREM/REM cycles.

Much experimental work has been dedicated to the affects of temperature on sleep patterns $[9,18,21,22,23,30,44]$. The strong relationship between thermoregulation and sleep observed under a variety of experimental conditions has led to the suggestion that thermoregulation may have a role in sleep regulation comparable to that of the circadian rhythm and homeostatic drive for sleep [17]. This suggests that, in the course of model development, responses to various temperature effects might be a good element to be considered both for its potential scientific insight as well as for the purposes of model validation. Also, the response to an impulsive stimulus interrupting sleep is a test of the model that could be applied to other mathematical models of sleep/wake regulation. Fulcher et al. [16] modeled the impact of impulsive stimuli such as loud sounds in a model of sleep/wake cycling without REM/NREM. Motivated by experiments, their model includes a varying arousal threshold for sensory stimuli that 
reaches a maximum near the middle of the night and then decreases; to our knowledge, a varying threshold for temperature deviations has not been reported. Initially when we interrupted sleep due to CBT deviations, the model tended to produce periods of wakefulness much longer than needed in order to restore body temperature (on the order of half an hour), due to strong inhibition from wake-active populations to POAH. Since the data show that these wake bouts are more commonly less than 10 minutes (See [18]), we changed the dynamics in the inhibitory connections from ORX and MRF to POAH so that the inhibition is weaker during sleep (see Table 3). Our implementation of thermoregulation is simple and phenomenological, yet gives good qualitative agreement with several features in data of temperature effects on sleep. The model presented here is a step towards testing that intriguing hypothesis. A more detailed model of temperature regulation might improve the quantitative agreement with data.

The model presented here considers the dynamics of the sleep/wake cycle including wakings from REM sleep due to the need for active thermoregulation in an environment away from thermoneutrality. Other important temperature effects have not been included here. We have not incorporated temperature effects on sleep latency or REM latency [18, 30]. Temperature effects on sleep latency could be incorporated by inhibiting transitions from wakefulness into NREM sleep at temperatures requiring efficient thermoregulation; the strengthening homeostatic sleep drive would in time overcome that inhibition. Mathematically, there are a number of possible implementations, but biological considerations should be foremost. Similarly, the model should sometimes thermoregulate through a prolonged NREM state rather than enter REM sleep. Another extension of the model would include warm sensitive neurons in POAH that sense distal skin temperatures. It has been reported that increasing the gradient of distal- toproximal skin temperatures (such as by foot warming) reduces sleep onset latency, and in fact Krauchi et al. [20] found that distal-to-proximal skin temperature gradient was a better predictor of sleep onset latency than CBT. These important effects will be addressed in future work.

It is worth mentioning that deterministic models constructed with coupled Morris-Lecar equations may exhibit more rigidity than is evident in human sleep data. While models of this class -including the present modelare quite robust to noise (results not shown), there are many variations in sleep patterns (durations of nights, durations of REM cycles, length of nighttime awakenings) that are difficult to reproduce with the general inclusion of noise. Thoughtful incorporation of stochastic elements might provide a more realistic range of sleep behaviors (such as variations in wake durations) without modulating the inhibitory connection to POAH. Firing rate models have also been used to model sleep/wake regulation and may be less rigid $[16,13]$.

The model presented here is intended to further the development of mathematical models for human sleep/wake regulation by incorporating 
thermoregulation and temperature effects, in accordance with their significance as observed in experiments. We have used the model to examine the impact of ambient temperature in limiting the duration of individual REM bouts and total time in REM sleep. Further modifications to our model would allow for the transition from REM sleep to NREM sleep, rather than always waking up due to temperature deviations. Whether the model transitions from REM to NREM sleep or from REM to the awake state may be dependent on the amount by which ambient temperature deviates from thermoneutral, as well as the current state of the circadian rhythm and sleep homeostat. Understanding the roles of CBT rhythm and thermoregulation in shaping the structure of a typical night's sleep and the variation that occurs on a particular night remain important challenges in sleep research.

5. Acknowledgements. The authors would like to thank the Institute for Mathematics and its Applications for hosting the Workshop for Women in Applied Mathematics (WhAM!) and the Mathematical Biosciences Institute (MBI) for hosting a working reunion. This work was in part supported by the Department of Defense (DoD) through the National Defense Science \& Engineering Graduate Fellowship (NDSEG) Program (PBP), the National Science Foundation (NSF) Graduate Research Fellowship under Grant No. DGE-1247271 (PBP), Research Training Groups under Grant No. DMS-1344962 (PBP), the MBI and the NSF under grant DMS-0931642 (JB), NSF CAREER Award DMS-0956057 (JB), the Alfred P. Sloan Foundation (JB), DGES grant MTM2012-31714 (GH), and "Juan de la Cierva" program (GH).

\section{REFERENCES}

[1] P. Achermann And A. Borbely, Simulation of Daytime Vigilance by the Additive Interaction of a Homeostatic and Circadian Process, Bio. Cybern., 71(2), (1994), pp. 115-121.

[2] E. Aserinsky, The maximal capacity for sleep: Rapid eye movement density as an indes of sleep satiety, Biological Psychiatry, 1(2), (1969), pp. 147-159.

[3] A. Bassi, E. Vivaldi and A. Ocampo-Garces, The Time Course of the Probability of Transition Into and Out of REM Sleep, Sleep, 32(5), (2009), pp. 655-669.

[4] J. Benington, Debating how REM Sleep is Regulated (and by what), J. Sleep Res., 11(1), (2002), pp. 29-33.

[5] R. Berger and N. Phillips, Sleep and Energy Conservation, Physiology, 8, (1993), pp. 276-281.

[6] V. Booth and C. Diniz Benn, Physiologically-Based Modeling of Sleep-Wake Regulatory Networks, Math. Biosci., 250, (2014), pp. 154-68.

[7] A. Borisyuk and J. Rinzel, Understanding Neuronal Dynamics by Geometrical Dissection of Minimal Models, in Methods and Models in Neurophysics, Les Houches Summer Schoool, Session LXXX, C. Chow, B. Gutkin, D. Hansel and C. Meunier (eds). Elsevier, (2005)

[8] R. Brown, R. Basheer, J. McKenna, R. Stecker and W. McCarley, Control of Sleep and Wakefulness, Physiol. Rev., 92(3), (2012), pp. 1087-1187. 
[9] C. Czeisler, E. Weitzman, M. Moore-Ede, J. Zimmerman and R. Knauer, Human Sleep: Its Duration and Organization Depend on its Circadian Phase, Science, 210(4475), (1980), pp. 1264-1267.

[10] C. Czeisler, J. Zimmerman, J. Ronda, M. Moore-Ede and E. Weitzman, Timing of REM Sleep is Coupled to the Circadian Rhythm of Body Temperature in Man, Sleep, 2(3), (1980), pp. 329-346.

[11] S. Dahn, D. Beersma And A. Borbely, Timing of Human Sleep: Recovery Process Gated by a Circadian Pacemaker, Am. J. Physiol., 246, (1984), pp. 161183.

[12] D. DiJk And C. Czeisler, Contribution of the Circadian Pacemaker and the Sleep Homeostat to Sleep Propensity, Sleep Structure, Electroencephalographic Slow Waves, and Sleep Spindle Activity in Humans, J. Neurosci., 15(5) (1995), pp. 3526-3538.

[13] C. Diniz Behn and V. Booth, Simulating Microinjection Experiments in a Novle Model of the Rat Sleep-Wake Regulatory Network, J. Neruophysiol., 103, (2010), pp. 1937-1953.

[14] C. Diniz Behn, A. Ananthasubramaniam and V. Booth, Contrasting Existence and Robustness of REM/Non-REM Cycling in Physiologically Based Models of REM Sleep Regulatory Networks, J. Appl. Dyn. Syst., 12(1), (2013), pp. 279-314

[15] P. Franken, Long-Term vs. Short-Term Processes Regulating REM Sleep, J. Sleep Res., 11(1), (2002), pp. 17-28.

[16] B. Fulcher, A.Phillips And A. Robinson, Modeling the Impact of Impulsive Stimuli on Sleep-Wake Dynamics, Phys. Rev. E., 78(5), (2008), pp. 051920.

[17] S. Gilbert, C. van den Heuvel, S. Ferguson and D. Dawson, Thermoregulation as a Sleep Signaling System, Sleep Med. Rev., 8(2), (2004), pp. 81-93.

[18] E. Haskell, J. Palca, J. Walker, R. Berger and H. Heller, Metabolism and Thermoregulation During Stages of Sleep in Humans Exposed to Heat and Cold, J. Appl. Physiol., 51, (1981), pp. 948-954.

[19] D. Kenshalo, J. Nafe And B. Brooks, Variations in Thermal Sensitivity, Science, 134(3472), (1961), pp. 104-105.

[20] K. Krauchi, C. Cajochen, E. Werth and A. Wirz-Justice, Functional Link Between Distal Vasodilation and Sleep-Onset Latency, Am. J. Physiol.-Reg. I., 278, (2000), pp. 741-748.

[21] K. Krauchi, The Human Sleep-Wake Cycle Reconsidered form a Thermoregulatory Point of View, Physiol. Behav., 90(2-3), (2007), pp. 236-245.

[22] K. Krauchi and T Deboer, The interrelationship between sleep regulations and thermoregulation, Front. in Biosci., 15, (2010), pp. 604-625.

[23] V. Kumar, Body Temperature and Sleep: Are they Controlled by the Same Mechanism?, Sleep Biol. Rhythms, 2(2), (2004), pp. 103-124.

[24] R. Kumar, A. Bose and B. Mallick, A Mathematical Model towards Understanding the Mechanism of Neural Regulations of Wake-NREM-REM States, PLoS ONE, 7(8), (2012).

[25] M. Kryger, T. Roth and W. Dement, Principles and Practice of Sleep Medicine, in Elsevier Health Sciences, (2010).

[26] J. Lu, A. Buorkum, M. Xu, S. Gaus, P. Shiromani and C. Saper, Selective Activation of the Extended Ventrolateral Preoptic Nucleus during Rapid Eye Movement Sleep, J. Neuro. Sci., 22(11), (2002), pp. 4568-4576.

[27] B. Mallick, A. Singh, And M. Khanday, Activation of Inactivation Process Initiates Rapid Eye Movement Sleep, Prog. Neurobiol., 97(3), (2012), pp 259276.

[28] H. Mallick and M. Kumar, Basal forebrain thermoregulatory mechanism modulates auto-regulated sleep, Front.in Neurology, 3, (2012).

[29] C. Morris And H. Lecar, Voltage Oscillations in the Barnacle Giant Muscle Fiber, Biophys. J., 35(1), (1981), pp. 193-213.

[30] A. Muzet, J. Ehrhart, V. Candas, J.P. Libert and J. J. Vogt, REM Sleep and 
Ambient Temperature in Man, Int. J. Neurosci., 18(1-2), (1983), pp. 117-125.

[31] M. Nakao, D. McGinty, R. Szymusiak and M. Yamamoto, A Thermoregulatory Model of Sleep Control, in Jpn. J. Physiol., 45(2), (1995), pp. 291-309.

[32] M. Nakao, D. McGinty, R. Szymusiak and M. Yamamoto, Dynamical Features of Thermoregulatory Model of Sleep Control, Jpn. J. Physiol., 45(2), (1995), pp. 311-326.

[33] M. Nakao, T. Ichikawa, D. McGinty, R. Szymusiak and M. Yamamoto, A Model-Based Prediction of Circadian System Responses to A Heat Load Pulse, in 18th Annual International Conference of the IEEE: Engineering in Medicine and Biology Society, 4, (1996), pp. 1729-1730.

[34] D. Ishiura, A. Karashima, N. Katayama and M. NakaO, Integrated Model Incorporating Circadian Phase Dynamics and Thermoregulatory Mechanism of Sleep, Sleep Biol. Rhythms, 5(4), (2007), pp. 259-270.

[35] F. Nijhout, J. Best and M. Reed, Escape from Homeostasis, to appear in Math. Biosci.

[36] D. Pal and B. Mallick, Neural Mechanism of Rapid Eye Movement Sleep Generations with Reference to REM-off Neurons in Locus Coeruleus, Indian J. Med. Res., 125(6), (2007), pp. 721-739.

[37] A. Phillips, P. Robinson and E. Klerman, Arousal State Feedback as Potential Physiological Generator of the Ultradian REM/NREM Sleep Cycle, J. Theor. Biol., 319(21), (2013), pp. 75-87.

[38] S. Quanten, E. de Valck, R. Cluydts, J.M. Aerts and, D. Berckmans, Individualized and Time-Variant Models for the Functional Link Between Thermoregulation and Sleep Onset, J. Sleep Res., 15(2), (2006), pp. 183-198.

[39] M. Rempe, J. Best and D. Terman, A Mathematical Model of the Sleep/Wake Cycle, J. Math Biol., 60(5), (2010), pp. 615-644.

[40] M. Rodriguez-Girones, Predicting Body Temperature of Endotherms During Shuttling, J. Therm. Biol., 27(5), (2002), pp.393-403.

[41] V. Savage and G. West, A Quantitative Theoretical Framework for Understanding Mammalian Sleep, Proceedings of the National Academy of Science, 104(3), (2007), pp.1051-1056.

[42] M. Schmidt, The Energy Allocation Function of Sleep: A Unifying Theory of Sleep, Torpor, and Continuous Wakefulness, Neuroscience and Biobehavioral Reviews, 47, (2014), pp.122-153.

[43] S. Strogatz, The Mathematical Structure of the Human Sleep-Wake Cycle, in Springer, Heidelberg (1986).

[44] J. Zulley, R. Wever, And J. Aschoff, The Dependence of Onset and Duration of Sleep on the Circadian Rhythm of Rectal Temperature, Eur. J. Physiol., 391(4),(1981), pp. 314-318. 